\title{
Contrast induced nephropathy - cardiologist perspective
}

Sanjib Kumar Sharma*, Laxman Dubey, Sogunuru Guruprasad, Gangapatnam Subramanyam

Department of Cardiology, College of Medical Sciences, Bharatpur, Nepal

\section{Citation \\ Sharma SK, Dubey L, Guruprasad S, et al. Contrast induced nephropathy-cardiologist perspective. Nepalese Heart Journal 2013;10(1):30-37.}

\section{Keywords}

cardiovascular intervention, Contrast induced nephropathy, contrast media

\begin{abstract}
Use of contrast media for cardiovascular intervention is associated with risk of contrast induced nephropathy. Contrast induced nephropathy is associated with increased morbidity, prolonged hospitalization, potential need for dialysis and increased mortality rate. Although the consequences of contrast induced nephropathy are well known, the prospective identification of patient at risk for nephropathy has been inconsistent. The mechanism of contrast induced nephropathy is complex and not fully understood. Direct tubular toxicity and disturbances of renal hemodynamic with altered glomerular filtration and renal medullary ischemia are the most important path-physiological mechanism. Prevention of contrast induced nephropathy is address in numerous studies. The most attractive agents include hydration, $\mathrm{N}$-acetylcysteine, and infusion of sodium bicarbonate. This review focuses on the definition, pathophysiology and prevention of contrast induced nephropathy.
\end{abstract}

and percutaneous coronary interventions (PCI) are associated with higher risk of CIN. ${ }^{2}$

\section{Definition of CIN:}

CIN is defined as a sudden decline in renal function occurring after exposure to intravenous radiographic contrast agents that is not attributable to other causes. Typically, the serum creatinine level begins

\footnotetext{
*Corresponding Author:

Sanjib Kumar Sharma

Department of Cardiology

College of Medical Sciences

Bharatpur, Nepal

Email: drsanjib@yahoo.com
} 
to increase 24 to 72 hours after administration of contrast medium, peaks at 3 to 5 days and requires further 3 to 5 days to return to baseline.

The Kidney Disease outcome Quality Initiative (K/DOQI)in 2012 guidelines defines $\mathrm{CIN}$ as a rise in serum creatinine of $\geq 0.5 \mathrm{mg} / \mathrm{dl}(\geq 44 \mathrm{mmol} / \mathrm{l})$ or a $25 \%$ increase from baseline value, assessed at 48 hours after a radiological procedure. ${ }^{5}$ This definition also consistently predicted major adverse cardiovascular events after PCI. ${ }^{6}$

\section{Epidemiology of CIN:}

The rate of incidence of CIN as a complication of radiographic diagnostic and interventional studies varies markedly depending on the definition used or on other variables such as the type of radiologic procedure performed, the dose and the type of contrast agent administered, the different patients population in regard to number and type of risk factors, and the length of patient follow-up. The incidence of CIN in general population without risk factors is reported to be $0.6-2.3 \% .^{7}$ However, it is important to recognize that the incidence of CIN in selected subjects is much higher, i.e., 9-40\% among diabetic patients with mild-to-moderate chronic renal insufficiency and 50-90\% with severe chronic renal insufficiency has been reported. ${ }^{89}$ In the Mayo Clinic intervention cardiology registry, the incidence of CIN was 3.3\% among 7586 patients. ${ }^{10}$ In high-risk patients the incidence of CIN has been calculated to be $>20 \%$ to $30 \%{ }^{11}$ Overall, when comparing the largest randomized controlled trials $(\mathrm{N} \geq 250)$ during period from 2007 to 2012, protocol defined CIN incidence ranged from $<1 \%$ to $>20 \%$ with an increase incidence after emergency PCI. ${ }^{12}$

\section{Pathophysiology of CIN}

The mechanism of CIN is complex and not fully understood. The most important pathophysiologic links for CIN identified so far include direct tubular toxicity and disturbances of the renal hemodynamic with altered glomerular function and renal medullary ischemia.

\section{a) Direct cytotoxic effect:}

Contrast media have a direct cytotoxic effect on renal structures, including reduction of transepithelial resistance, insult permeability, polarized cellular enzyme release and other parameters of renal tubular cell viability. ${ }^{13}$ In vitro studies of proximal tubular cells incubated with contrast media demonstrated altered cellular metabolism pathologic changes consistent with toxicity and intracellular enzyme release. ${ }^{14}$ Patients who have received radio contrast material have been noted to have an increased urinary excretion of lysosomal enzymes and small molecular weight proteins, which are nonspecific indicators of tubular toxicity. ${ }^{15}$ The direct renal tubular cytotoxicity is suggested by histological changes such as cell injury and the presence of enzymuria following contrast administration. ${ }^{16}$ An increased production of oxygen free radicals was documented in an experimental model of CIN ${ }^{17}$ According to this finding, oxidant-mediated injury has been suggested as a mechanism of cytotoxic effect in the pathogenesis of CIN. Yoshioka et al. ${ }^{18}$ found that contrast agents can reduce the activity of antioxidant enzyme catalase and superoxide dismutase in the renal cortex of volume depleted rats.

\section{b) Renal Hemodynamics (vasoconstrictive effects on renal blood flow)}

In addition to these direct tubular effects, radio contrast agent may induce a biphasic hemodynamic response, with an initial brief period of vasodilation, followed by a variable period of renal vasoconstriction. ${ }^{19}$

Weiberg et al. ${ }^{20}$ demonstrated that all patients have an early initial increase in renal flow after radio contrast administration. Surprisingly, in contrast to non-diabetic patients, diabetic patients with a lower baseline renal blood flow manifest an earlier, more sustained and more pronounced increase in renal blood flow after contrast injection. ${ }^{20}$ The mechanism by which contrast medium causes subsequent vasoconstriction is still not fully understood. Alteration in the metabolism of prostaglandin, nitric oxide, endothelin, or adenosine possibly plays a role. ${ }^{21}$ Barkris et al. ${ }^{22}$ found a reduced Glomerular filtration rate (GFR) after the administration of a dopamine-1 receptor antagonist and an improvement by using the selective dopamine-1 receptor agonist fenoldopam. Interestingly, the use of vasodilators such as dopamine and atrial natriuretic peptide (ANP) may actually exacerbate medullary ischemia by causing redistribution of blood flow from the medulla to the cortex.

Although the mechanisms are not fully understood, medullary hypoxia and tubular collapse with occlusion is thought to be important in the pathogenesis of CIN.

\section{c) Vasoactive Substances in the Pathogenesis of CIN}

The release of endothelin and vasopressin, along with a reduction in prostacyclin synthesis and release, reduces blood flow to anoxic medulla. ${ }^{13}$ Endothelin, a strong endogenous vasoconstrictor, may contribute to the pathogenesis of CIN. After exposure to contrast material, the level of serum endothelin in animal models and in humans increases and is especially higher in patients with diabetes mellitus or impaired renal function. ${ }^{23,24}$ 


\section{d) Impaired Nitric oxide production and Vasodilatation}

Nitric oxide (NO) is a potent vasodilator produced from L-arginine by the enzyme NO synthase. In an in vitro study of cultured smooth muscle cells from the renal artery, Ribeiro and Colleagues 25 examined the effect of different contrast agents on NO production. The reduction in NO production was proportional to the osmolarity of the solution. Iodixanol (290 mOsm) was the only contrast agent that did not alter the production of NO. These observations suggest that, in addition to direct vasoconstriction of renal vessels, iodinated contrast agents also block an important pathway for vasodilatation and autoregulation. ${ }^{25}$ However, the effects of NO inhibition have not been confirmed in human studies.

\section{e) Reperfusion and Reactive Oxygen Species}

The intense vasoconstriction and loss of autoregulatory capacity can contribute to additional renal injury through the release of reactive oxygen species (eg, superoxide [OH]). Organ injury can occur when hypo perfusion of tissues generates reactive oxygen species that exceed the antioxidant reserve of the patient. The ability to accommodate oxidant injury decreases with age and is thought to contribute to the increased risk of CIN among older patients. Moreover, increased oxidative stress is present in chronic renal failure ${ }^{26}$ and in diabetes. ${ }^{27}$ It contributes to enhanced basal vascular tone and to impaired endothelium-dependent relaxation in chronic kidney disease. There are few data on the role of reactive oxygen species in the pathogenesis of CIN. In a study of oxidant injury following contrast injection, Sandhu and associates ${ }^{29}$ measured the increases in urinary malondialdehyde-to-creatinine ratio as a marker of oxidative stress. The malondialdehydeto-creatinine ratio increased following contrast infusion, suggesting a link between contrast infusion and free radical generation. In a study investigating the effects of cardiac catheterization on the generation of reactive oxygen species, Fiaccadori and colleagues ${ }^{30}$ measured urine and plasma levels of 3-nitrotyrosine(a marker of peroxynitrite generation from superoxide).Urinary 3-nitrotyrosine peaked after catheterization, but plasma levels remained elevated for up to 72 hours. However, in 193 lowrisk patients with normal renal function studied prospectively, there was no significant difference in the baseline serum levels of antioxidant compounds between those who did or did not develop renal failure after cardiac catheterization. ${ }^{31}$

\section{Prognosis of CIN}

The recovery from CIN is very likely and dialysis is infrequently required. The acute kidney injury seen in CIN is generally non-oliguric and reversible.
In high-risk patients, oliguria may develop within 24 hours of contrast medium administration. Currently, CIN is one of the most common causes of acute renal failure among hospitalized patients. Several studies demonstrated the close relationship between CIN and prognosis after PCI.,31 The development of CIN has been associated with an increase in morbidity and both inhospital and long-term mortality. In a retrospective study, Levy et al. ${ }^{5}$ concluded that patients who developed CIN had higher mortality (34\%) compared with patients (7\%) who did not develop CIN after contrast administration $(p<0.001$, odds ratio 5.5). In another study, Guberget al. ${ }^{4}$ studied the effects of contrast administration on morbidity and mortality in 439 patients with a baseline creatinine $>1.8 \mathrm{mg} / \mathrm{dl}$. The inhospital mortality rate was $22.6 \%$ for those requiring hemodialysis as a result of contrast administration. The cumulative 1-year mortality rate was $45.2 \%$ for those who required dialysis. Iakovou et al. ${ }^{32}$ reported that patients with CIN versus those without CIN had significantly elevated rates of hospitalization $(4.7 \%$ vs. $0.9 \%$, respectively) and 1 -year mortality $(32.3 \%$ vs. $13.9 \%)$. In a study of McCullough et al. ${ }^{3}$ acute renal failure requiring dialysis after coronary angioplasty was $1 \%$, and creatinine clearance, diabetes and contrast dose were shown to be independent predictors of acute renal failure requiring dialysis. The inhospital mortality for those developed acute renal failure was 35.7\% and the 2-year survival was $18.8 \%$. According to the result of Rihal and coworkers ${ }^{10}$ inhospital mortality in patients undergoing PCI and developing CIN was $22 \%$ versus only $1.4 \%$ in patients without CIN. Furthermore, among hospital survivors with acute renal failure, 1 - and 5-year estimated mortality rate was $12.1 \%$ and $44.6 \%$, respectively.

\section{Prevention of CIN}

Several studies have been performed to prevent CIN. The most attractive agents includes hydration, $\mathrm{N}$-acetylcysteine (NAC), and infusion of sodium bicarbonate. Others like dopamine, fenoldopam, theophylline, diuretics, atrial natriuretic peptide (ANP), calcium channel blockers, endothelin antagonist, and prophylactic hemodialysis are mostly found not to be useful hence not discussed here.

\section{Hydration}

Adequate hydration is the simplest and most effective way of protecting renal function. Currently hydration is the only universally accepted method to prevent CIN. ${ }^{21,33,34}$ Intravenous hydration seems better than oral hydration. When the patient is well hydrated, it appears more likely that renal medullary perfusion is increased due to the inhibition of vasopressin and the reduction of fluid viscosity 
of contrast media in the distal portion of tubular system. ${ }^{35}$ Many studies have demonstrated the benefits of hydration in preventing CIN. Solomon et al. $^{33}$ randomized 78 patients who underwent cardiac angiography to $0.45 \%$ saline only $(1 \mathrm{ml} / \mathrm{kg}$ body weight $/ \mathrm{h}$ ), mannitol with saline, or furosemide with saline. Among the patients, $11 \%$ in the salineonly group, $28 \%$ in the mannitol with saline group, and $40 \%$ in the furosemide with saline group developed CIN. The authors suggested that saline was beneficial in preventing CIN. In most studies, a uniform protocol with half-isotonic $(0.45 \%)$ saline at a rate of $1 \mathrm{ml} / \mathrm{kg} / \mathrm{h}$ before and after contrast exposure was employed..$^{36,37,38}$ Mueller et al. ${ }^{39}$ performed a randomized comparison of 2 hydration regimens (isotonic versus half-isotonic) in 1620 patients undergoing coronary angiography. CIN occurred in $0.7 \%$ of the patients with $0.9 \%$ saline versus $2.0 \%$ of those with half-isotonic saline $(p=0.04)$. The predefined subgroups benefited in particular from isotonic hydration: women, patients with diabetes and those receiving prevention of CIN. In another study, Taylor et al. ${ }^{40}$ tested the efficacy of outpatient oral pre-catheterization hydration (oral hydration with $1000 \mathrm{ml}$ clear liquid over $10 \mathrm{~h}$ ) followed by $6 \mathrm{~h}$ of intravenous hydration $(0.45 \%$ saline solution at $300 \mathrm{ml} / \mathrm{h}$ ) beginning just before contrast material exposure and compared this protocol with overnight intravenous hydration $(0.45 \%$ normal saline solution at $75 \mathrm{ml} / \mathrm{h}$ for $12 \mathrm{~h}$ before and after catheterization). The authors concluded that a hydration strategy compatible with outpatient cardiac catheterization was as effective as the traditional pre and post catheterization intravenous hydration protocol but was associated with a decrease in length of stay in hospital.

Brown et al. ${ }^{41}$ also found the benefits of hydration in preventing CIN in patients with serum creatinine concentration $\geq 2.0 \mathrm{mg} / \mathrm{dl}$. The disadvantages of hydration include its unsuitability for patients with cardiac failure and its limited use in emergency situation resulting from its requirement of fluid administration for several hours before contrast medium exposure. ${ }^{42}$ Based on the above evidence, all patients undergoing contrast-related procedure should receive adequate hydration. The most widely accepted protocol is administering $0.45 \%$ saline at 1 to $1.5 \mathrm{ml} / \mathrm{kg} / \mathrm{h}$ beginning $6-12 \mathrm{~h}$ prior to the procedure and continuing for up to $12 \mathrm{~h}$ following contrast administration..$^{21,33,36,43}$ Current K/DOQI Guideline on prevention of CI-AKI suggests a "good" urine output $(>150 \mathrm{ml} /$ hour $)$ in the first 6 hours of radiological procedures, which may reduces rate of AKI. In order to achieve urine flow rate of at least $150 \mathrm{ml} /$ hour, $>1.0-1.5 \mathrm{ml} / \mathrm{kg} /$ hour of intravenous fluid had to be administered for 3-12 hours before and 6-12 after contrast-media exposure. ${ }^{5}$

\section{N-Acetylcysteine}

NAC is an antioxidant and scavenger of oxygen free radical. It also increases the biogenic effect of NO by combining with NO to form S-nitrosothiol, which is a more stable and potent vasodilator than NO. It also increases the expression of NO synthase and may thus also improve blood flow. Based on the theory that CIN is caused primarily by reactive oxygen species, Tepel et al. ${ }^{44}$ compared the oral administration of the antioxidant NAC (600 mg twice a day on the day before and the day of examination) plus standard hydration to hydration alone in 83 patients undergoing computer tomography with intravenous administration of $75 \mathrm{ml}$ of nonionic, low-osmolality contrast agent. A significantly lower-incidence of CIN in the NAC group (2\%) was observed compared to the placebo group (21\%, $\mathrm{p}=0.01$ ). Baker et al. ${ }^{45}$ randomized 80 patients with stable renal dysfunction undergoing cardiac catheterization and intervention to a rapid protocol of intravenous NAC. CIN occurred in $5 \%$ in the NAC group and in $21 \%$ in the hydration group ( $p=$ $0.045)$. The study concluded that the administration of infusion NAC should be considered in all patients to preclude adequate oral prophylaxis, provided the patient is able to tolerate this degree of volume loading.

A protective effect of high dose (1200 mg twice daily) versus a standard dose (600 mg twice daily) along with saline hydration was also reported. ${ }^{46}$ In a cohort of 224 patients with chronic renal insufficiency (creatinine $>1.5 \mathrm{mg} / \mathrm{dl}$ or creatinine clearance $<60 \mathrm{ml} / \mathrm{min}$ ), CIN occurred in $11 \%$ of patients in the standard dose group and in $3.5 \%$ in the high dose group $(p=0.04)$. In the subgroup with the contrast dose $\geq 140 \mathrm{ml}$, CIN was more frequent in the standard group $(18.9 \%)$ than in the high dose group $(5.4 \%, \mathrm{p}=0.04)$, whereas no difference was found in the low-dose $(<140 \mathrm{ml})$ subgroup. Although several studies showed a protective effect, others demonstrated that oral administration of NAC does not protect renal function; particularly when moderate to high dose of contrast medium are used. ${ }^{27,47,48,49}$ Allaqband et al. ${ }^{47}$ randomized 123 patients to either saline alone or saline plus NAC at a dose of $600 \mathrm{mg}$ orally on the day before and after the day of procedure: no significant difference in CIN was observed between the NAC and the saline-only group. In a trial by Boccaluandro et al. ${ }^{49}$, the incidence of CIN in patients with chronic renal insufficiency (creatinine clearance $<50 \mathrm{ml} / \mathrm{min}$ ) undergoing cardiac catheterization was $13 \%$ in the NAC group (600 mg twice daily for $48 \mathrm{~h}$ starting the day before the procedure) and $12 \%$ in the control group $(p=0.84)$. Both groups received intravenous hydration $(75 \mathrm{ml} / \mathrm{h}$ of $0.45 \%$ saline solution for $24 \mathrm{~h}$ starting $12 \mathrm{~h}$ before the procedure). The study 
concluded that NAC with a intravenous fluid is as effective as fluid alone in the prevention of CIN when moderate to high doses of contrast media are used in patients with chronic renal insufficiency.

A meta analysis to access the efficacy of NAC in preventing CIN was performed by Pannu et al..$^{50}$, who reviewed 15 studies in NAC effect. The analysis indicates a significant heterogeneity in NAC effect among studies. NAC may reduce the incidence of CIN, but this finding is of borderline statistical significance, and there is significant heterogeneity among trials. In conclusion, NAC may be recommended for patients receiving lower doses of contrast, but its role in higher-risk population needs to be further investigated. If NAC is to be used as a preventive measure, it should be given at a dose of $600 \mathrm{mg}$ oral bid (1200 $\mathrm{mg}$ bid if creatinine $>2.5$ $\mathrm{mg} / \mathrm{dl}$ ) on the day before and day of the procedure. In addition, adequate hydration should be given at a rate of $1 \mathrm{ml} / \mathrm{kg} / \mathrm{h}$ for 6 to $12 \mathrm{~h}$ prior to contrast and up to $12 \mathrm{~h}$ following contrast administration. The current K/DOQI guideline suggest oral NAC, together with intravenous isotonic crystalloid, in patients at increased risk of contrast-induced acute kidney injury (CI-AKI). ${ }^{5}$

\section{Sodium Bicarbonate}

Experimental studies have demonstrated that pretreatment with sodium bicarbonate is more protective than sodium chloride in animal models of acute ischemic renal failure. ${ }^{51}$ Formation of free radical is promoted by an acid environment but inhibited by increasing $\mathrm{PH}$ of normal extracellular fluid, with the use of bicarbonate. ${ }^{52}$ The protective effect results from antioxidant effects and scavenging reactive free radical but not from better volume expansion in comparison with saline solution infusion. A prospective single-center randomized study of 119 patients by Merternet al. ${ }^{52}$ has suggested that the use of sodium bicarbonate hydration is superior to sodium chloride hydration. The most recent and probably the most complete systematic review ${ }^{53}$ analyzed MEDLINE, PubMed, EMBASE, and the Cochrane Central Register of Controlled Trials from 1950 to December 2008; conference proceedings; and ClinicalTrials.gov, without language restriction. This systematic review included RCTs of intravenous sodium bicarbonate that pre-specified the outcome of CI-AKI as a $25 \%$ increase in baseline serum creatinine concentration or an absolute increase of $0.5 \mathrm{mg} / \mathrm{dl}$ after contrastmedia administration. Twenty-three published and unpublished trials with information on 3563 patients and 396 CI-AKI events were included. The pooled RR was 0.62 (95\% CI 0.45-0.86), with evidence of significant heterogeneity across studies. Some heterogeneity was due to the difference in the estimates between published and unpublished studies: RR 0.43 (95\% CI $0.25-0.75)$ vs. 0.78 (95\% CI 0.52-1.17), respectively. Meta-regression showed that small, poor-quality studies that assessed outcomes soon after contrast-media administration were more likely to suggest the benefit of bicarbonate $(\mathrm{P}<0.05$ for all $)$. No clear effects of treatment on the risk for dialysis, heart failure, and total mortality were identified. Although confirmation in a larger multicenter study is necessary, infusion of sodium bicarbonate may provide a simple, safe and effective method for the prevention of CIN. K/DOQI 2012 guideline recommends intravenous volume expansion with either isotonic sodium chloride or sodium bicarbonate solution in patients at increased risk of CIN. ${ }^{5}$

\section{Management of CIN and Therapeutic Recommendation}

$\mathrm{CIN}$ is an iatrogenic disorder and the major cause of inhospital renal failure and contributes to overall morbidity and mortality. In most cases, the functional impairment is reversed within 1 or 2 weeks and the need for dialysis is rare. There is no specific therapy for the treatment of CIN. Prevention of CIN relies on careful procedure selection and patient assessment. Patients with underlying renal insufficiency and a history of diabetes represent the highest risk population. Potential nephrotoxic agents should be withdrawn at least $24 \mathrm{~h}$ before contrast exposure, low osmolar contrast agent or iso-osmolar should be used when possible, the total dose of contrast media should be minimized and repeated contrast administration within a short period of time should be avoided. Patients should have their renal function checked by serum creatinine before and at 48 to $72 \mathrm{~h}$ after contrast administration. All patients undergoing angiography should receive adequate hydration. Guidelines ${ }^{5,54}$ recommended intravenous isotonic saline or sodium bicarbonate $1.5 \mathrm{ml} / \mathrm{kg} /$ hour or more, starting 3-12 $\mathrm{h}$ before the procedure. The post procedure hydration target is a urine output of $150 \mathrm{ml} /$ hour and to achieve urine output $150 \mathrm{ml} /$ hour intravenous administration of sodium bicarbonate or normal saline at $150 \mathrm{ml} /$ hour for at least six hours is required. Although there are many new promising modalities in the prevention of CIN, such as $\mathrm{NaHCO}$ and hemofiltration, hydration remains the most effective methods of prevention. Patients with chronic renal insufficiency receiving large contrast dose $(>140 \mathrm{ml})$ may also be given high-dose NAC (2 X $1200 \mathrm{mg})$. 


\section{REFERENCE:}

1. Hou SH, Bushinksy DA, Wish JB, et al. Hospital-acquired renal insufficiency: a prospective study. Am J Med. 1983; 74:243248.

2. Nask K, Hafeez A, Hous S. Hospital acquired renal insufficiency. Am J Kidney Dis 2002;39:930-936.

3. Guitterez NV, Wolyn R, Rocher LL, et al. Acute renal failure after coronary intervention: incidence, risk factors and relationship to mortality. Am J Med 1997; 103:368-375.

4. Gruberg L, Mintz GS, Mehran R, et al. The prognostic implications of further renal function deterioration with $48 \mathrm{~h}$ of interventional coronary procedures in patients with pre-existent chronic renal insufficiency. J Am CollCardiol 2000; 36: 1542-1548.

5. Contrast-induced AKI: definition, epidemiology, and prognosis. Kidney International Supplements 2012;2:69-88.

6. Schwann NM, Horrow JC, Strong MD, $3 \mathrm{rd}$, et al. Does off-pump coronary artery bypass reduce the incidence of clinically evident renal dysfunction after multivessel myocardial revascularization? AnesthAnalg 2004; 99: 959-964.

7. Lasser EC, Lyon SG, Berry CC. Reports on contrast media reactions: analysis of data from reports to the US Food and Drug Administration. Radiology 1997; 203: 605610.

8. Harkonen S, Kjellstrand CM. Exacerbation of diabetic renal failure following intravenous pyelography. Am J Med 1977; 63:939-946.

9. Manske CL, Sprafka JM, Strony JT, et al. Contrast nephropathy in azotemic diabetic patients undergoing coronary angiography. Am J Med 1990; 89: 615-620.

10. Rihal CS, Textor SC, Grill DE et al. Incidence and prognostic importance of acute renal failure after percutaneous coronary intervention. Circulation 2002; 105: 22592264.

11. Tapel M, Aspelin P, Lemeire N. Contrast induced nephropathy: A clinical and evidence based approach. Circulation 2006; 113:17991806.

12. Perrin T, Descombes E, Cook S. Contrastinduced nephropathy in invasive Cardiology: Incidence, pathophysiology, diagnosis, prevention and prognosis. Swiss Med Wkly 2012; 142:w13608.

13. Rudnick MR. Contrast-induced nephropathy. Pathophysiology and strategies for prevention. J Invasive Cardiol 2004; 16(suppl: 7s-11s).

14. Humes HD, Hunt DA, White MD. Direct toxic effect of the radiocontrast agent diatrizoate on renal proximal tubule cells. Am J Physiol 1987; 252: F246-F255.

15. Waybill MM, Waybill PN. Contrast mediainduced nephrotoxocity: identification of patients at risk and algorithms for prevention. J VascintervRadiol 2001; 12:3-9.

16. Rudnick MR, Berns JS, Cohen RM, et al. Contrast media-associated nephrotoxocity. SeminNephrol 1997; 17:15-26.

17. Bakris $\mathrm{GL}$, Lass $\mathrm{N}$, Gabber $\mathrm{AO}$, et al. Radiocontrast medium-induced declines in renal function: a role for oxygen free radicals. Am J Physiol 1990; 258(1 pt 2):F115-F120.

18. Yoshioka T, Fogo A, Beckman JK. Reduced activity of antioxidant enzymes underlies contrast media-induced renal injury in volume depletion. Kidney Int 1992; 41:10081015.

19. Barrett BJ. Contrast nephrotoxicity. J Am SocNephrol 1994; 5:125-137.

20. Weisberg LS, Kurnik PB, Kurnik BRC. Risk of radiocontrast nephropathy in patients with and without diabetes mellitus. Kidney Int 1994; 45:259-265.

21. Murphy SW, Barrett BJ, Parfrey PS. Contrast nephropathy. J Am SocNephrol 2000; 11:177182.

22. Bakris GL, Lass NA, Glock D. Renal hemodynamic in radiocontrast medium induced renal dysfunction: a role for dopamine-1 receptors. Kidney Int 1999; 56:206-210.

23. Clark BA, Ducksoo K, Epstein FH. Endothelin and atrial natriuretic peptide levels following radiocontrast exposure in humans. Am J Kidney Dis 1997; 30:82-86.

24. Heyman CN, Clark BA, Kaiser N, et al. Radiocontrast agents induced endothelin release in vivo and vitro. J Am SocNephrol 1992; 3:58-65.

25. Ribeiro L, de Assuncao e Silva F, Kurihara $\mathrm{RS}$, et al. Evaluation of the nitric oxide production in rat renal artery smooth muscle cells culture exposed to radiocontrast agents. Kidney Int2004;65:589-596. 
26. Massy ZA, Nguyen-Khoa T. Oxidative stress and chronic renal failure: markers and management. J Nephrol2002; 15:336-341.

27. Scott JA, King GL. Oxidative stress and antioxidant treatment in diabetes. Ann N Y AcadSci2004; 1031:204-213.

28. Schnackenberg CG. Oxygen radicals in cardiovascular-renal disease. CurrOpinPharmacol2002; 2:121-125.

29. Sandhu C, Newman DJ, Morgan R, et al. The role of oxygen free radicals in contrast induced nephrotoxicity. AcadRadiol2002; 9:S436 -S437.

30. Fiaccadori E, Maggiore U, Rotelli C, et al. Plasma and urinary free 3-nitrotyrosine following cardiac angiography procedures with nonionic radiocontrast media. Nephrol Dial Transplant 2004; 19:865-869.

31. Best PJ, Lennon R, Ting HH, et al. The impact of renal insufficiency on clinical outcome in patients undergoing percutaneous coronary interventions. J Am Coll. Cardiol 2002; 39: 1113-1119.

32. Iakovou I, Dangas G, Lansky AJ, et al. Incidence, predictors and economic impact on contrast induced nephropathy: result in 8628 patients treated with percutaneous coronary interventions (abstract). J Am CollCardiol 2002; 39:2A.

33. Solomon R, Werner C, Mann D, et al. Effects of saline, mannitol and furosemide to prevent acute decrease in renal function induced by radiocontrast agents. N Engl J Med 1994; 331:1416-1420.

34. Trivedi HS, Moore H, Nasr S et al. A randomized prospective trial to access the role of saline hydration on the development of contrast nephrotoxicity. Nephron ClinPract 2003; 93:C29-C34.

35. Persson PB, Patzak A. Renal haemodynamic alternation in contrast medium nephropathy and the benefit of hydration. Nephrol Dial Transplant 2005;20 (suppl):i2-i5.

36. Goldnberg I, Shechter M, Matetzky S, et al. Oral acetylcysteine as an adjunct to saline hydration for the prevention of contrastinduced nephropathy following coronary angiography. Eur Heart J 2004; 25:211-8.

37. Shyu KJ, Cheng JJ, Kuan P. Acetylcysteine protects against acute renal damage in patients with abnormal renal function undergoning a coronary procedure. J Am CollCardiol 2002; 40: $1383-8$.
38. Briguori C, Colombo A, Violante A, et al. Standard vs double dose of n-acetylsysteine to prevent contrast agent associated nephrotoxicity. Eur Heart J 2004; 25:206-11.

39. Mueller C, Buerkle G, Buettner HJ. Prevention of contrast media-associated nephropathy: randomized comparison of 2 hyfration regimens in 1620 patients undergoing coronary angioplasty. Arch Intern Med 2002; 162:329-336.

40. Taylor AJ,Jotchkiss D, Morse RW, et al. PREPARED Preparation for Angiography in Renal Dysfunction: a randomized trial of inpatients vs outpatients hydration protocols for cardiac catheterization in mild-tomoderate renal dysfunction. Chest 1998; 114 : 1570-1574.

41. Brown RS, Ransil B, Clark BA. Prehydration protects against contrast nephyrpathy in high risk patients undergoing cardiac catheterization (abstract). J Am SocNephrol 1990; 1: 330 .

42. Erley CM. Does hydration prevention radiocontrast-induced acute renal failure? Nephrol Dial Transplant 1999; 14: 1064-1066

43. Agrawal M, Stouffer GA. Contrast induced nephropathy after angiography. Am Med Sci2002; 323: 252-258.

44. Tepel M, van der Giet M, Schwarzfeld C, et al. Prevention of radiographic contrastagent induced reduction in renal function by acetylcysteine. N Engl J Med 2000; 343:180184.

45. Baker CS, Wragg A, Kumer S et al. A rapid protocol for the prevention of contrastinduced renal dysfunction: the RAPPID study. J Am CollCardiol2003; 41:2114-2118.

46. Kay J, Chow WH, Chan TM, et al. Acetylcysteine for prevention of acute deterioration of renal function following elective coronary angiography and intervention: a randomized controlled. J A M A 2003, 289: 553-558.

47. Allaqaband S, Tumuluri R, Melik AM, et al. Prospective randomized study of $\mathrm{N}$-acetylcysteine, fenoldopam and saline for prevention of radiocontrast-induced nephropathy. CathetCardiovascIntervent 2002; 57:279-283.

48. Briguori C, Manganelli F, Scarpato P, et al. Acetylcysteine and contrast agent associated nephrotoxicity. J Am CollCardiol 2002; 40:298-303. 
49. Boccalandro F, Amhad M, Smalling RW, et al. Oral acetylcysteine does not protect renal function from moderate to high doses of intravenous radiographic contrast. CathetCardiovascIntervent 2003； 58:336341.

50. Pannu N, Manns B, Lee H, Tonelli M. Systematic review of the impact of $\mathrm{N}$-acetylcysteine on contrast nephropathy. Kidney Int 2004; 65:1366-137.

51. Atkins JL. Effect of sodium bicarbonate preloading on ischemic renal failure. Nephron 1986; 44:70-74.
52. Mertern GJ, Burgess WP, Gray LN, et al. Prevention of contrast-induced nephropathy with sodium bicarbonate: a randomized controlled trial. J A M A 2004; 291:23282334.

53. Zoungas S, Ninomiya $\mathrm{T}$, Huxley $\mathrm{R}$, et al. Systemic Review: sodium bicarbonate treatment regimens for the prevention of contrast-induced nephropathy. Ann Intern Med 2009; 151:631-638.

54. Thomsen HS. Guideline for contrast media from the European Society of Urogenital Radiology. Am J Roentgenol 2003; 181:14631471. 